(Aus dem pharmakologischen Institute der Universität Breslau.)

\title{
Beiträge zur Lehre von der Diurese.
}

\author{
XIII. \\ Über die Wirkung des Suprarenins auf die Harnsekretion. \\ Von \\ Dr. Joh. BiberfeId, \\ Privatdozent und Institutsassistent.
}

\begin{abstract}
Über die Wirkung des in den Nebennieren gebildeten Körpers auf die Niere und ihre Tätigkeit ist in der Literatur wenig zu finden. Gottlie $b^{1}$ ) hat überlebende Nieren mit Nebennierenextrakt durchströmt und beobachtet, dass dann die Gefässe sich verengten, auch wenn sie vorher durch Chloralhydrat zur Erweiterung gebracht waren. In Beziehung auf eine Beeinflussung der Nierensekretion habe ich nur eine Publikation finden kömmen; E. Bardier und H. Frenkel ${ }^{2}$ ) haben Hunden Nebennierenextrakt in travenös beigebracht. Etwa 1 Minute nach Beendigung der Infusion beobachteten sie eine Verminderung der Urinmenge ${ }^{3}$ ), die durchschnittlich 3 Minuten dauerte, und an die sich eine Vermehrung anschloss. In Versuchen, in denen sie den allgemeinen Blutdruck und das Volumen der Niere-massen, fanden sie, dass die Kurven des Blutdruckes und der Harnmenge im umgekehrten Sinne verliefen, während Nierenerweiterung und Diurese stets zu gleicher Zeit eintraten. Aus diesem Befunde schliessen zu wollen, was übrigens die Verfasser nicht ausdrücklich tun, dass die Vergrösserung des Nierenvolumens die Ursache der Diurese sei; ist logisch nicht zulässig. Denn erstens setzt ein solcher Schluss voraus, dass die Volumenvergrösserung nur durch eine Erweiterung der

1) Arch. f. exp. Pathol. u. Pharmakol. Bd. 43 S. 289.

2) E. Bardier u. H. Frenkel, Journ. de physiol. et de pathol. génér. 1899 p. 950 .

3) Manchmal blieb zu Anfang die Urinmenge gleichgross.
\end{abstract}


Blutgefässe hervorgebracht werde, und eine solche Voraussetzung ist nicht ohne weiteres berechtigt, da die bei fast jeder Art von Diurese beobachtete Zunahme des Nierenvolumens, wie schon von anderer Seite hervorgehoben worden ist, zum grössten Teil durch die pralle Anfüllung der Harnwege mit Flüssigkeit hervorgerufen wird. Zweitens aber ist, selbst wenn man eine stärkere Blutdurchströmung als erwiesen annimmt, damit natürlich noch nicht gezeigt, dass diese auch die Ursache der Diurese sei. Gefässerweiterung und vermehrte Durchströmung in der Niere brauchen nur die gleiche Bedeutung zu haben wie derselbe Vorgang bei anderen Organen, auch nicht drüsigen, z. B. Muskel, Gehirn (Anämie während des Schlafes, gute Durchströmung beim Wachen). Wie wohl noch niemand auf den Gedanken gekommen ist, die stärkere Durchblutung des Muskels als Ursache seiner Zusammenziehung aufzufassen, ebensowenig ist ein ähnlicher Schluss für die Verhältnisse bei der Nierensekretion berechtigt. In beiden Fällen ist die bessere Versorgung mit Blut nur eine, allerdings fast stets notwendige Begleiterscheinung der anderweitig irgendwie verursachten Tätigkeitssteigerung.

Gelegentlich von Versuchen, die ich über die Frage anstellte, ob subkutane Suprarenininjektionen Albuminurie erzeugen, sah ich, dass solche Injektionen regelmässig eine Diurese hervorriefen. Eine genauere Verfolgung dieses Befundes schien mir lohnend, besonders unter dem Gesichtspunkte, wie sich der Kochsalzgehalt des diuretischen Harnes verhielte, da ich bei einer andersartigen, ebenso wie die Suprarenindiurese mit Glykosurie verbundenen Diurese der nach Phlorhizin - gefunden hatte, dass das $\mathrm{NaCl}$ nur in Spuren im Urin vorhanden war.

Im Anhang füge ich die Protokolle meiner Versuche bei. Bei den an Kaninchen angestellten Versuchen $1,2,4,5,6,7,14,15$, 16 ist jede andere Beeinflussung als durch die Suprarenininjektion vermieden worden. Die folgende Tabelle gibt die hier erzielten Resultate wieder.

Aus dieser Zusammenstellung erhellt, dass nach der subkutanen Injektion von Suprarenin in der Dosis von 1,5-2,5 mg pro kg Kaninchen regelmässig in der Zeit von $1 / 2-1$ Stunde eine starke Diurese auftritt; ihr Maximum erreicht diese meist in der Zeit zwischen der 1. und 2. Stunde. Die Dauer der Diurese ist recht erheblich: mit Ausnahme des ersten Versuches erstreckt sie sich in allen über mehr als 5 Stunden. 
Zeitlicher Verlauf der Suprarenindiurese nach der subkutanen Injektion.

\begin{tabular}{|c|c|c|c|c|}
\hline $\begin{array}{c}\text { Ver- } \\
\text { suchs- } \\
\text { nummer }\end{array}$ & Beginn & Dauer & Maximum & $\begin{array}{c}\text { Dosis pro } \\
\text { Kilo- } \\
\text { gramm }\end{array}$ \\
\hline $\begin{array}{r}1 \\
2 \\
4 \\
5 \\
6 \\
7 \\
14 \\
15 \\
15 \\
16\end{array}$ & 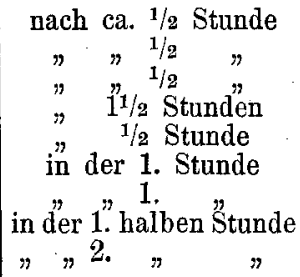 & 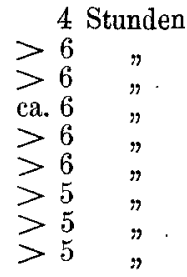 & $\begin{array}{ccc}\text { nach } & 1^{1 / 2}-2^{1 / 2} & \text { Stunden } \\
" & 1-2 & " \\
" & 1-1^{1 / 2} & " \\
" & 2-3^{1 / 2} & " \\
" & 1-3 & " \\
" & 1-1^{1 / 2} & " \\
" & 1 / 2-1^{1 / 2} & " \\
" & 1 / 2-1 & \text { Stunde } \\
" & 2-2^{1 / 2} & \text { Stunden }\end{array}$ & $\begin{array}{ll}2^{1 / 2} \mathrm{mg} \\
2 \quad " \\
2 & " \\
2 & " \\
2 & " \\
1,66 " & \\
2 & " \\
2 & " \\
1,72 & \end{array}$ \\
\hline
\end{tabular}

Gehen wir auf die Frage ein, in welcher Weise wir uns das Zustandekommen der Diurese vorzustellen haben, so liegt es nahe, eine Abhängigkeit von der dem Suprarenin eigentümlichen Beeinflussung der Blutströmung $\mathrm{zu}$ vermuten. Ich habe deswegen, trotzdem es bereits bekannt war, dass subkutan beigebrachtes Suprarenin den Blutdruck wenig beeinflusst, die Blutdruckkurve von einigen Kaninchen aufgenommen, denen ähnliche Mengen Suprarenin, wie die genannten, eingespritzt worden waren. Wie die Protokolle der Versuche 11,12,13,18, 19 zeigen, lässt sich aus der Höhe des Druckes, die zu der Zeit des gewöhnlichen Beginns der Diurese und weiterhin verzeichnet ist, keinerlei Beziehung ableiten. Der Blutdruck ist $\mathrm{zu}$ dieser Zeit manchmal etwas höher, meist etwas niedriger als vor der Injektion, hält sich jedoch fast stets in den Grenzen der Schwankungen, die man auch sonst bei Kaninchen zu sehen gewohnt ist, besonders, wenn die Tiere längere Zeit aufgebunden liegen bleiben. Jedenfalls dürfen wir nach dem ganzen Verlauf der Kurve eine wesentliche Erweiterung der Nierengefässe nicht annehmen, da eine solche sicherlich zu einer viel tieferen Senkung des Druckes Anlass gegeben hätte, zumal ja dann voraussichtlich auch die Gefässe der anderen Abdominalorgane die gleiche oder wenigstens eine ähnliche Erweiterung unter dem Einflusse des Giftes erlitten haben würden. - Auch die ausnehmend lange Dauer der Diurese spricht an sich schon gegen die Annahme einer Abhängigkeit von Änderungen der Blutdurchströmung.

Aus den Protokollen über die letztgenannten Versuche ist ersichtlich, dass in ihnen keine oder nur eine ganz geringe Diurese 
vorhanden war. Der Grund dieses von dem sonst Beobachteten abweichenden Verhaltens wurde zuerst in der geänderten Lagerung der Tiere (Rückenlage, während die Tiere sonst meist frei in Käfig gelassen und nur zum Katheterisieren aufgebunden wurden) und in der dadurch eventuell bedingten Abkühlung gesucht. Doch liess sich bald zeigen, dass diese Vermutung nicht richtig war; weder das Aufgebundensein während des ganzen Versuches, noch relativ starke Abkühlung (Versuche 15 und 16) brachten eine Änderung des Diureseverlaufes hervor. Es bleibt nach meiner Meinung nichts übrig, als die Operation (Finführung der Manometerkanüle in die Karotis) bezw. den damit verbundenen, wenn auch sehr geringen Blutverlust als Hindernis für das Zustandekommen der Diurese anzusprechen. Michaud ${ }^{1}$, ein Schüler von L. Asher, hat nachgewiesen, dass schon die Entziehung einer kleinen Menge von Blut selbst eine starke Theophyllindiurese zum Aufhören bringen kann. Noch in einer anderen Beziehung verliefen die Blutdruckversuche abweichend von den übrigen. Nur in einem einzigen von den fünf angestellten war im Urin Zucker (mit der Trommer'schen Probe) nachweisbar, und auch da nur innerhalb einer relativ kurzen Zeit, während in keinem der anderen Kaninchenversuche Zucker vermisst wurde. Einen Grund für das Ausbleiben der Glykosurie bei den an das Kymographion gelegten Tieren vermag ich nicht anzugeben; die Kaninchen waren anscheinend in demselben Ernährungszustande wie die anderen und hatten das gleiche Futter bekommen. Jedenfalls legt dieses eigentümliche Verhalten den Gedanken nahe, einen Zusammenhang darin zu suchen, dass man die Zuckerausseheidung als die Ursache der Diurese ansieht. Eine gewisse Wahrschẹinlichkeit gewinnt dieser Gedanke durch die Resultate, die ich bei Hunden mit Suprarenin erzielte. Bei diesen babe ich nach Suprarenininjektion niemals eine so starke Diurese erhalten wie bei Kaninchen (Versuche 3, 9, 10, 17, 20). In den Versuchen 3, 10 und 20 ist aber doch eine Zunahme der ausgeschiedenen Harnmenge deutlich erkennbar, und zwar tritt diese erst nach längerer Zeit post injectionem auf als bei Kaninchen, und jedesmal ist in der ersten vermehrten Harnportion zum ersten Male Zucker nachweisbar ${ }^{2}$ ). -

1) Über das Scheidevermögen der Niere bei'Blutentzug und über die Wirkungsweise der Dinretika. Zeitschr. f. Biol. Bd. 46 S. 198.

2) Bei den Kaninchen war dies nicht immer der Fall. (Versuch 4.) 
In den beiden anderen Hundeversuchen (9 und 17) ist keine Vermehrung, einmal sogar eine Verminderung zu sehen gewesen, und hier fehlte während der ganzen Versuchsdauer Zucker im Harn.

Überall ist der Kochsalzgehalt der einzelnen Urinportionen ermittelt worden ${ }^{1}$ ). Bei einem grossen Teil der Kaninchenversuche war auch hier eine Zeit zu finden, während der, ähnlich wie ich es für die Phlorhizindiurese beschrieben habe ${ }^{2}$ ), der Kochsalzgehalt auf ein Minimum zurückging, so dass er nicht mehr quantitativ bestimmbar war (Versuche 1, 2, 5, 7, 14, 19); auch hier wurde wenigstens einmal dieses Stadium erst erreicht, als die Diurese bereits vorbei war. In anderen Fällen (z. B. 15, 16), ist eine so erhebliche Verminderung des CaCl-Gehaltes trotz reichlicher Diurese nicht zu konstatieren, sondern nur das gewöhnliche, dem Anwachsen des Harnflusses entsprechende Sinken der Konzentration. Andererseits ist ein fast völliges Verschwinden des Kochsalzes auch da vorgekommen, wo gar keine Diurese erzielt worden ist (Kymographionversuch 19). An Hunden habe ich nur einmal (Versuch 10) einen fast kochsalzfreien Harn erhalten, während die Phlorhizinversuche ausschliesslich an Hunden angestellt waren und fast stets das erwähnte Resultat ergaben.

Weshalb nun das Suprarenin die Kochsalzausscheidung in so eigenartiger Weise beeinflusst, ist kaum zu sagen. Für die analoge Wirkung des Phlorhizins habe ${ }^{3}$ ) ich kürzlich folgendes als eventuellen Grund angesprochen. In der Norm werde Zucker deshalb nicht von der Niere sezerniert, weil der Blutzucker, soweit er von den Epithelien der Tubuli contorti aufgenommen sei, dort auch sofort als leicht benutzbares Brennmaterial aufgebraucht werde; Phlorhizin

1) Einen Teil der Chloranalysen hat Herr Dr. chem. Klose ausgeführt; ieh bin ihm für seine Freundlichkeit zu Dank verpflichtet.

2) Pflüger's Arch. Bd. 112 S. 398. Dieser Befund steht im tatsächlichen Gegensatze zu der Behauptung O. Loewi's, dass bei der Phlorhizindiurese die Menge des ausgeschiedenen $\mathrm{NaCl}$ sich nicht ändere. L oewi hatte hieraus einen Beweis für die Filtrationshypothese konstruiert. Ich habe nun 1, c. gezeigt, dass selbst, wenn seine Behauptung richtig wäre, doch der Kochsalzgehalt in seinen Versuchen eher für die Sekretionstheorie spräche. Wie Herr Prof. Asher mich brieflich aufmerksam machte, hat er bereits vor mir (Arch. f. Biol. Bd. 46 S. 66 ff.) die Prozentzahlen in Loewi's Versuchen aus dessen Angaben berechnet und daraus die Unrichtigkeit von $L_{o}$ ewi's Beweisführung gefolgert. Die betreffende Stelle war mir leider entgangen.

3) Zentralbl. f. d. ges. Physiol. u. Pathol. des Stoffwechsels 1907 Nir. 9 u. 10. 
lähme nun die Oxydationskraft der betreffenden Zellen und verursache dadurch gewissermaassen indirekt eine Glykosurie. Der nicht verbrannte und darum zur Ausscheidung kommende Zucker wirke aber als Reiz auf die Tubuluszellen und führe somit zur Diurese; diese gibt dann aber keine Veranlassung zur Mebrausfuhr von $\mathrm{NaCl}$, da für eine solche in der Blutzusammensetzung kein Grund vorliege und der "diuretische" Reiz sich eben nur auf die Beschaffung von Lösungswasser für den Zucker erstrecke. - Diese Vermutung ist für die Suprarenindiurese nicht anwendbar, da hier nach den Angaben in der Literatur der Blutzuckergehalt vergrössert ist; bei einer Hyperglykämie wird aber sonst keine solche Armut an Kochsalz im Urin gefunden.

Im Hinblick auf frühere Erfahrungen ${ }^{1}$ ) habe ich in einem Versuche ein Kaninchen durch geeignete Fütterung wasserreich gemacht und habe dann nachgesehen, wie sich die Kochsalzausfuhr unter der Suprareninwirkung gestaltete (Versuch 8). Es liess sich kein wesentlicher Unterschied gegen das sonstige Verhalten erkennen: der schon vor der Suprarenineinspritzung geringe Gehalt an $\mathrm{NaCl}$ sank noch mehr.

Bemerkenswert ist vielleicht noch, dass anscheinend Morphin sowohl beim Hunde als auch beim Kaninchen (Versuch 3, 9, 17, 21) einen die Suprarenindiurese hemmenden Einfluss besitzt.

Im Anschluss an die vorstehende Mitteilung sei es mir gestattet, auf einige Punkte aus früheren Publikationen dieser Reihe einzugehen, die von anderen Autoren zum Gegenstand der Kritik gemacht worden sind.

In Nr. X dieser Beiträge ${ }^{2}$ ) hatte ich berichtet, dass bei Kaninchen, die mit Ferrocyannatrium angereichert waren, und denen ich Eisenchloridlösung an die Nieren heranbrachte, der so in der Niere entstandene Niederschlag von Berlinerblau niemals in dem Kapselr a u me der Glomeruli zu sehen war, trotz Blaufärbung der Glomeruluskapillaren; dagegen waren die Niederschläge in den Zellen und im Lumen der Tub. cont. vorhanden. Ich schloss daraus, dass an dieser Stelle sicherlich beide Substanzen $\left(\mathrm{Na}_{4} \mathrm{FeCy}_{6}\right.$ und $\left.\mathrm{Fe}_{2} \mathrm{Cl}_{6}\right)$ sezerniert seien, dass aber wahrscheinlich beide, mindestens aber der eine die

1) Ruschhaupt,.Pflüger's Arch. Bd. 91 S. 597. Biberfeld, Pflüger's Arch. Bd. 112 S. 403.

2) Pflüger's Arch. Bd. 105 S. 308. 
Epithelschicht des Glomerulus nicht passiert habe. - Basler ${ }^{1}$, der später ebenfalls die Berlinerblauniederschläge untersuchte, findet diesen Schluss merkwürdig, „denn die wenigen blauen Punkte, die man ab und zu auf den Biberfeld'schen Abbildungen zwischen den Umrahmungen der Harnkanälchen sieht und über deren Lage sich gar nichts Bestimmtes sagen lässt, sind meiner Auffassung nach nicht beweisend." - Ferner wendet Basler gegen die Richtigkeit des von mir gezogenen Schlusses ein, dass in meinen Versuchen die Nierenarterie behufs Einfübrung der für die Eisenchloridinjektion gebrauchten Kanüle abgebunden und dadurch die Niere von der normalen Zirkulation ausgeschaltet worden sei; eine solche Niere sei das gleiche wie die Niere eines soeben getöteten Tieres. Ausserdem habe das Eisenchlorid „eine derartig ätzende Wirkung, dass in jeder Zelle, zu der es gelangt, dass Leben sofort vernichtet wird. Aus diesem Grunde kann von einer physiologischen Ausscheidung des Eisenchlorids durch die Niere... überhaupt nicht die Rede sein, sondern das Eisenchlorid wird einfach durch die Gefässe hindurchgepresst."

Um mit dem letzten Einwand zu beginnen, so erscheint die Logik Basler's nicht einwandfrei. Selbst wenn meine Versuche in der Weise, wie Basler es sich denkt, angestellt worden wären, so wird nach meiner Meinung nur der Schluss um so zwingender, dass in den Glomerulis keine körperfremden Substanzen ausgeschieden werden, da ja sogar unter Bedingungen, die sicherlich dort ein Durchtreten von sonst zurückgehaltenen Substanzen, z. B. Eiweiss, begünstigen, doch kein Durchtreten von Ferrocyannatrium und Eisenchlorid zu konstatieren war. Die Glomeruhuskapsel passieren also wahrscheinlich weder die Cyanverbindung noch das Eisenchlorid oder mindestens eins von beiden nicht. Nun ist ja Ferrocyannatrium im Urin nachgewiesen, und da auch B asler kaum annehmen wird, dass die Niere Eisenchlorid (z. B. per os gegebenes) unter keinen Umständen herausschaffen könne, so bleibt eben nichts anderes übrig als mindestens für eins der beiden Salze eine andere Stelle als Sekretionsort anzusprechen, d. h. eben den Tubulus contortus; da es aber ferner kaum möglich sein dürfte, einen plausiblen Grund dafür ausfindig zu machen, dass die beiden Salze an verschiedenen Stellen sezerniert werden, so ist der Schluss berechtigt, dass beide in den Tubulis contortis herausbefördert werden.

1) Pflüger's Arch. Bd. 112 S. 217. 
Aber tatsächlich habe ich mich gerade bemüht, durch meine Versuchsmethodik Einwänden wie denen Basler's zu begegnen, und habe dies auch betont. Ich habe das Eisenchlorid nicht in die Nierenarterie, sondern in die Aorta gespritzt: "wenn die Sekretion gut im Gange war, eröffnete ich die Bauchhöhle, führte eine Kanüle in die Aorta bis zur Höhe der linken Nierenarterie und spritzte durch diese Kanüle das. Fällungsmittel ein. Der normale Blutstrom durch die Niere war so niemals ganz unterbrochen" (1. c. S. 310). Ferner habe ich ausdrücklich angegeben, dass ich sehr dünne Eisenchloridlösungen genommen habe; ich habe $2 \mathrm{ccm}$ des offizinellen Liquor ferri sesquichlorati und $1 \mathrm{ccm}$ stark verdünter $\mathrm{HCl}$ auf $100 \mathrm{ccm}$ Wasser genommen und diese Mischung eingespritzt. Da der Liquor $10 \%$ Eisen enthält, hatte die injizierte Flüssigkeit eine Konzentration von etwa $0,5-0,6 \%$ Eisenchlorid; die von $\mathrm{B}$ asler als selbstverständlich vorausgesetzte ätzende Wirkung der Eisenchloridlösungen beginnt aber erst bei einem Gehalt von ca. $5 \% 0^{1}$ ). - Ich glaube deshalb zu der Behauptung berechtigt zu sein, dass die von mir beobachtete Sekretion der körperfremden Salze durchaus in physiologischer Weise zustande gekommen ist.

Wenn nun Basler meint, dass die wenigen Punkte in meinen Abbildungen, über deren Lage man nichts aussagen könne, nichts beweisen, so muss ich gestehen, dass ich das nicht begreife. Klarere Bilder als z. B. die der Stellen $a$ in Fig. I $b$ Taf. V, wo ganze Strecken der Tubuli contorti mit Niederschlägen angefüllt erscheinen, sind nach meiner Meinung kaum zu liefern. Möglicherweise wären sie noch deutlicher geworden, wenn ich eine stärkere Vergrösserung reproduziert hätte. Dies habe ich nicht getan, da ich dann den Zeiss'schen Zeichenapparat nicht hätte benutzen können, und es mir gerade auf eine objektive Nachbildung ankam.

Möglichst günstige Bedingungen für eine Filtration im Glomerulus hatten wir in den Versuchen hergestellt, über die in Nr. XI dieser Beiträge berichtet ist ${ }^{2}$ ). Wir hatten eine Kanüle in die Aorta unterhalb der linken Nierenarterie eingebunden, dann die Aorta oberhalb der rechten Nierenarterie abgeklemmt und sofort unter einem dem Blutdrucke entsprechenden (resp. etwas höheren) Drucke eine echte Lösung eines Farbstoffes durch die Kanüle gespritzt; der

1) Filehne, Arzneimittellehre S. 203.

2) Wilh. Filehne u. Joh. Biberfeld, Pflüger's Arch. Bd. 111 S. 1. 
Farbstoff wurde mit Alkohol gefällt. Die Niederschläge hatten wir, trotzdem die Glomeruluskapillaren mit ihnen angefüllt waren, niemals im Kapselraume gefunden. Wir sahen diesen Befund als einen Beweis dafür an, dass im Glomerulus eine Filtration überhaupt nicht stattfände. Dieser Schluss war nun so ohne weiteres nicht zwingend. Denn wir hatten tatsächlich nur nachgewiesen, dass das gelöste Karmin nicht durchgedrungen war, damit aber natürlich noch nicht den Beweis geliefert, dass auch keine "Flüssigkeit" (in unserem Falle das Lösungswasser) passiert sei. Zu der Erweiterung unserer Folgerung wurden wir verleitet durch die Ergebnisse unserer Versuche, Wasser durch eine homogene Membran (Gelatinemembran) hindurchzusaugen, die alle ein negatives Resultat gegeben hatten. Diese Versuche litten aber an einem technischen Fehler: wir hatten den zum Saugen notwendigen negativen Druck durch eine Wasserstrahlpumpe erzeugt, dadurch aber anscheinend an der unteren Fläche der Gelatinemembran jedes durchtretende Tröpfchen zum Verdampfen gebracht, so dass es zu keiner Flüssigkeitsansammlung kam. Benutzt man einen positiven Druck, so kann man schon bei relativ kleinen Druckhöhen Flüssigkeit durch die Membran hindurchbringen ${ }^{1}$ ). Eine Filtration ist also auch durch homogene Membranen möglich. Trotzdem bleibt aber doch unser Befund, dass gelöstes Karmin die Epithelschicht ni cht passiert hat, beweisend dafür, dass nicht alle Stoffe, die in der auf dem Glomerulus lastenden Flüssigkeit gelöst sind, mit dieser Flüssigkeit die Glomeruluswand durchdringen; hierzu gehört noch, dass die betreffende Substanz sich in der Membran löse. Wenn also E. Frey ${ }^{2}$ ). einwendet, dass unsere Verwahrung gegen die Annahme einer Filtration in der Niere gewissermaassen nur ein Streit um den Ausdruck sei, man brauche dann nur zu sagen, durch die Filtration lösen sich eben die Substanzen in der Glomerulusmembran, so ist das keineswegs richtig. Wie ich an anderer Stelle ausgeführt habe ${ }^{\mathbf{3}}$ ), ist die Löslichkeit eines Stoffes in der Glomerulusmembran geradezu das Ausschlaggebende für die Möglichkeit seines Austritts an dieser Stelle. Von den Vertretern der Filtrationshypothese ist es der sogenannte Sekretionstheorie stets zum Vorwurf gemacht worden, sie schreibe dem Glomerulus mehr Kraftleistung zu, als er mit Hilfe des Blutdruckes entfalten könne, wenn sie annehme, dass im Glomerulus

1) Vgl. auch Hertz, Zaitschr. f. physiol. Chemie Bd. 48 S. 347.

2) E. Frey, Pflüger's Arch. Bd. 115 S. 117.

3) Zentralbl. f. d. ges. Physiol. u. Pathol. d. Stoffw. 1907 Nr. 9 u. 10. 
Wasser und Salze von den übrigen im Blutwasser gelösten Bestandteilen getrennt werde; hierzu seien mehr als $80 \mathrm{~cm}$ Druck nötig, während der vorhandene (= Blutdruck) nur ca. $8 \mathrm{~cm}$ betrage. Dieser an sich berechtigte Einwand wird aber hinfällig, wenn man auf chemische Umsetzungen als Kraftquellen rekurriert. Die Wandung des Glomerulus legt chemisch die betreffenden Substanzen (die "spezifischen" Harnbestandteile und wahrscheinlich auch einen grossen Teil der Salze) in reversibler Bindung fest. Ich möchte hierfür folgendes Analogon anführen: Denken wir uns eine homogene Membran, die mit einer schwach sauren NaCl-Lösung getränkt sei; auf diese drücke eine schwach ammoniakalische Silbersalzlösung, und zwar so, dass die Silberlösung sich stets erneuere (Vorbeiströmen). Hier wird sich in den obersten Schichten der Membran ein Niederschlag von $\mathrm{AgCl}$ bilden, der später durch die vorbeipassierende ammoniakalische Flüssigkeit wieder gelöst und mit fortgeführt werden wird. Durch die Membran hindurch wird aber, solange genügend $\mathrm{NaCl}$ bei saurer Reaktion vorhanden ist, sicher nur Wasser dringen, ohne dass der von der Flüssigkeit ausgeübte Druck so gross zu sein braucht, wie er sein müsste, wenn durch eine mechanische Kraft das Wasser von dem gelösten Bestandteil getrennt werden soll. Und analoge Verhältnisse kann man sich, ohne "vitale" Zelleigenschaften in Anspruch zu nehmen, im Glomerulusepithel vorwaltend denken; z. B. könnten die Eiweisskörper, vermöge ihrer Zusammensetzung aus sauren und basischen Komplexen, im Zustande der Dissoziation befindliche Salze temporär festlegen, und Ähnliches mehr.

Das ist nun alles sicherlich eine vorläufig durch nichts als höchstens durch Analogien mit anderen Drüsen gestützte Vermutung, aber da bis jetzt, soweit ich sehe, auch nichts gegen sie spricht, so ist es doch wohl erlaubt, sie zur "Erklärung" der Nierentätigkeit mit heranzuziehen. Jedenfalls ermöglicht sie es, uns eine "mechanistische" Vorstellung von der Glomerulusfunktion $\mathrm{zu}$ bilden, die die Annahme entbehrlich macht, es fände in den Tubulis contortis eine Rückresorption von Wasser und gelösten Bestandteilen statt, eine Annahme, die die Vertreter der Filtrationshypothese zwingt, den Zellen der Tubuli contorti neben Sekretionseigenschaften auch noch die Fähigkeit zu vindizieren, vermöge "vitaler" Kapazität zu resorbieren. Denn physikalisch-chemisch ist, wie Tamann sagt, eine Rückresorption undenkbar.

Eine Möglichkeit physikalischer Auffassung der Rückresorption 
scheint uns E. Fre ${ }^{1}$ ) zu bieten. In sehr exakten Versuchen bemüht er sich, Aufschluss über die "physikalische" Leistung der Niere zu gewinnen. Er unterscheidet scharf zwischen dieser und der chemischen Arbeit der Niere; die letztere, die Entfernung der harnfähigen Substanzen aus dem Organismus, ist nach ihm einer mechanistischen Auffassung nicht zugänglich; dagegen wird die physikalische, die er in der Änderung der Gesamtkonzentration des Harnes gegenüber der des Blutes sieht, in folgender Weise geleistet: Wird z. B. nach Infusion einer konzentrierten Salzlösung ein bochgestellter Urin von der Niere geliefert, so ist dies dadurch zustande gekommen, dass der Ureterdruck (der hier grösser sei als der Blutdruck im zweiten Kapillarsystem) Wasser aus dem "provisorischen Harne" (= Glomerulusfiltrat der anderen Autoren) in die umspinnenden Kapillaren hineinpresse. Umgekehrt werde bei der Wasserdiurese Wasser mechanisch dureh den Blutdruck aus dem Blute der umspinnenden Kapillaren in die Harnwege hinausgepresst; in diesem Falle habe sich der Blutdruck ungefähr in gleicher Höhe vom Glomerulus auf das zweite Kapillarsystem fortgepflanzt. „Eine weitere Erklärung für die Ursache dieser beiden Mechanismen hat sich nicht ergeben." (Bd. 115, S. 179.)

Auf die vielen interessanten und wertvollen Einzelheiten der Frey'schen Arbeiten möchte ich hier nicht eingehen, sondern nur auf zwei Punkte hinweisen, von denen der eine für Frey's Auffassung nicht gerade wesentlich ist, aber doch wegen der Wichtigkeit der behaupteten Tatsache Besprechung verdient; der gegen den zweiten Punkt zu machende Einwand ist allerdings, wie mir scheint, von grundsätzlicher Bedeutung.

Fre ${ }^{2}$ ) gibt an, dass er nach dem Einspritzen konzentrierter Lösungen (Kochsalz, Glaubersalz, Harnstoff) stets eine annähernd gleiche Konzentration des Harnes gefunden habe; diese entsprach ungefähr einer Gefrierpunktsdepression von $1,2^{\circ}$. Frey ist anscheinend geneigt, hierin eine Gesetzmässigkeit zu erblicken. Dies ist jedoch nicht der Fall, sondern der durch Infusion von konzentrierten Salzlösungen gelieferte Urin zeigt unter Umständen einen viel höheren Gehalt. So hat der verstorbene Institutsassistent Dr. Ruschhaupt bei nicht veröffentlichten Versuchen über die Frage, welches die höchste erreichbare Kochsalzkonzentration im

1) Pflüger's Arch. Bd. 112 S. 71 u. Bd. 115 S. 179 ff.

2) Bd. 112 S. 78 ff. 
Urin sei, NaCl-Werte von $3 \%$ und darüber erzielt. Ein solcher $\mathrm{NaCl}$-Gehalt würde aber allein schon (ohne $\stackrel{+}{U}$ usw.) eine Depression von zirka $1,8^{\circ}$ ergeben. Ferner finde ich bei A. Loeb ${ }^{1}$ ), dass beim Kaninchen $\mathrm{NaCl}-$ Konzentrationen bis zu 3,8\%, also entsprechend $\triangle=$ mehr als $-2,0$ beobachtet worden sind, und ähnliche Ergebnisse liessen sich zweifellos in der Literatur noch oft finden. - Das eigenartige, von Frey gefundene Resultat darf also nicht als allgemein gültig angesehen werden.

Das zweite Bedenken, dem ich Ausdruck geben möchte, ist folgendes: Selbst wenn man die Frey'sche Voraussetzung zugeben wollte, dass einmal im Ureter (Salzdiurese), das andere Mal (Wasserdiurese) im zweiten Kapillarsystem ein Druck ungefähr gleich dem Blutdruck im Glomerulus bestände, so wäre doch dieser niemals gross genug, um mechanisch Wasser aus dem Blut, resp. dem provisorischen Harne abzupressen. Denn der Blutdruck in der Arteria renalis hat nach Frey (Bd. 112, S. 106) nur ungefähr eine Höhe von $8 \mathrm{~cm} \mathrm{Hg}$, während, um reines Wasser aus dem Blute abzupressen, ein Druck von zirka 7 Atmosphären nötig ist ${ }^{2}$ ); auch um aus dem Glomerulusfiltrat Wasser abzupressen, bedarf es eines fast eben so bohen Druckes, denn dieses besitzt ja gerade nach der Filtrationshypothese den gleichen osmotischen Druck wie das Blut minus dem minimalen Druck der Eiweisskörper. Wenn Frey in seiner $\mathrm{Zu}$ sammenfassung (S. 126) schreibt: „Es wird durch den geringen Überdruck auf der Harnseite der Harnkanälchen ein allmähliches Wasserabpressen aus ihnen hinaus in das Blut stattfinden " usw., so widerspricht dies, wie mir scheint, den allgemein gültigen Anschauungen von den osmotischen Druckkräften; sollte aber wirklich (wofür allerdings nichts anzuführen sein dürfte) in der Niere schon ein so geringer Druck fähig sein, das Lösungsmittel von dem gelösten Stoffe zu trennen, dann ist nicht einzusehen, warum nicht das gleiche schon im Glomerulus möglich sein sollte. Es wäre dann der oben erwähnte, auch neuerdings (vergl. Metzner, Nagel's Handbuch der Physiologie Band II, 1, Seite 235) als wesentlich angeführte Einwand gegen die Hei denhain'sche Theorie hinfällig ${ }^{3}$ ), selbst wenn man die zur Produktion eines von den spezifischen Be-

1) Arch. f. exp. Pathol. u. Pharmak. Bd. Ј̆4 S. 344.

2) Vgl. Metzner, Nagels Handbuch der Physiol. Bd. 2, 1, S. 235.

3) Und dies um so mehr, als zum Abpressen von Wasser und Salzen nur ca. $80 \mathrm{~cm} \mathrm{Hg}$ notwendig sind. 
standteilen und eines grossen Teiles der Salze freien Urins nötigen Kräfte nicht - wie angedeutet - in chemischen Umsetzungen suchen will.

Frey (Bd. 112 S. 85 und an anderen Stellen) hält es, ebenso wie andere Autoren, für selbstverständlich, dass, „sollte wirklich die Nierenzelle z. B. durch Ausstossen einer mit einem Stoffe beladenen Vakuole einen Überschuss an festen Stoffen in den Harn bringen, so stellt sich sogleich eine Konzentrationsänderung ein, sobald der Stoff in ,Lösung' geht, und ein Wasserstrom setzt ein, der die Konzentration auszugleichen bestrebt ist. Hier müssen wieder physikalische Kräfte vorhanden sein, um dies zu verhindern". Ich glaube, einen solehen Zwang braucht man keineswegs anzuerkennen. Man schreibt einem Exkretionsorgane, wie es doch die Niere ausschliesslich ist, wohl kaum zu viel "vitalistische" Eigenschaften zu, wenn man supponiert, dass sie im Laufe der Entwicklung die Fähigkeit erworben hat (z. B. durch reichliche Ansammlung von Lipoiden an dem Aussenrand der Zellen), für Wasser nur in der Richtung von innen nach aussen, nicht aber umgekehrt, durchgängig zu sein. Ähnliche Mechanismen, "Gefälle“ für Wasser nur von innen nach aussen, sind wohl sicher auch bei anderen Drüsen, die eine starke Sekretion von wechselnder Zusammensetzung aufweisen, z. B. Leber, Schweissdrüsen usw., vorhanden; in mancher Beziehung fungiert auch die Froschhaut in dieser Richtung.

Eine Nötigung, osmotischen Druckausgleichungen eine wesentliche Rolle bei der Harnbereitung zuzuerkennen, liegt demnach nicht vor.

Protokolle.

1. Kaninchen, männlich, 2500 g. 10. April 1906.

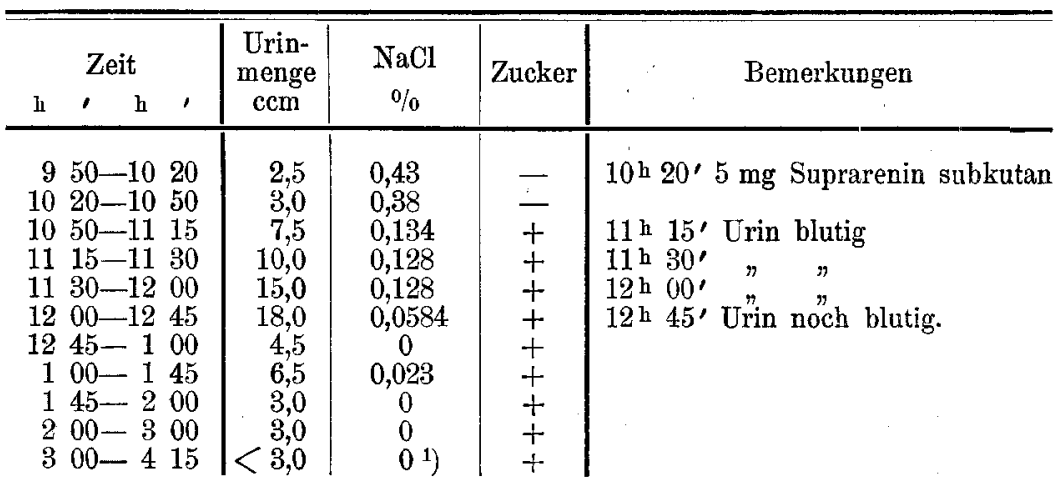

1) D. h. nur eben bemerkbare Opaleszenz bei Zusatz der Sibernitratlösung. 
2. Kaninchen, männlich, 1600 g. 27. April 1906.

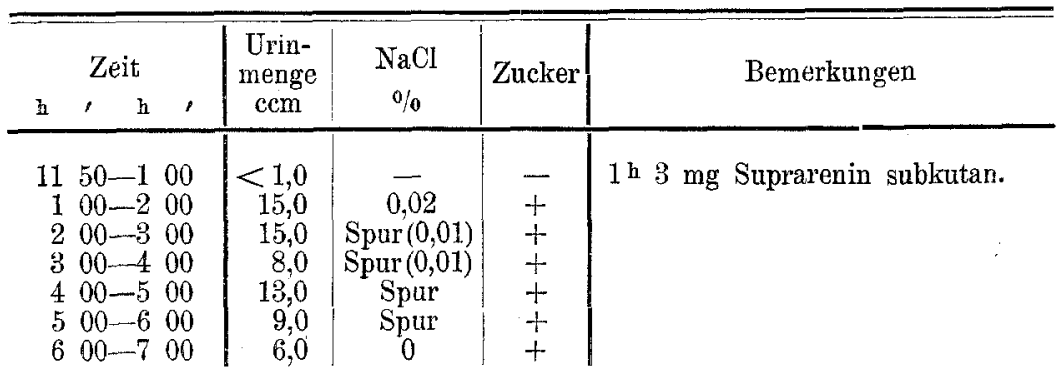

3. Hund, männlich, 12100 g. 28. April 1906.

\begin{tabular}{|c|c|c|c|c|}
\hline $900-1000$ & 2,5 & 0.29 & - & 9 h $0,02 \mathrm{~g}$ Morph. hydrochl. subkut. \\
\hline $1000-1100$ & 4 & 0,35 & - & $10 \mathrm{~h} 5.7 \mathrm{mg}$ Suprar. hydrochl. sub- \\
\hline $1100-1200$ & 12,0 & 0,29 & + & kutan; 11 h $5 \mathrm{mg}$ Suprar. boric. \\
\hline $1200-100$ & 14,0 & 0,128 & + & subkut.; $11 \mathrm{~h}$ Urin stark blutis \\
\hline $100-200$ & 19,0 & 0,047 & + & 12h Urin ganz schwach blutig. \\
\hline $200-300$ & 24,0 & 0,047 & + & \\
\hline $300-400$ & 20,0 & 0,07 & + & \\
\hline $400-5$ & 11,0 & 0,14 & + & \\
\hline
\end{tabular}

4. Kaninchen, männlich, 3000 g. 1. Mai 1906.

\begin{tabular}{rrr|r|r|r|rr}
10 & $30-11$ & 00 & 2,0 & 0,234 & - & 11 h 6 mg Suprarenin subkutan. \\
11 & $00-11$ & 30 & 6,5 & 0,438 & - & \\
11 & $30-12$ & 00 & 11,0 & 0,47 & - & \\
12 & $00-12$ & 30 & 23,0 & 0,26 & + & \\
12 & $30-1$ & 00 & 13,0 & 0,35 & + & \\
1 & $00-2$ & 00 & 13,0 & 0,26 & + & \\
2 & $00-3$ & 00 & 11,0 & 0,09 & + & \\
3 & $00-4$ & 00 & 6,0 & 0,11 & + & \\
4 & $00-5$ & 00 & 15,0 & 0,15 & - &
\end{tabular}

5. Kaninchen, männlich, $2100 \mathrm{~g}$.

\begin{tabular}{rrr|r|r|r|rr}
9 & $45-10$ & 15 & 1,8 & 0,52 & - & $10 \mathrm{~h} 15^{\prime}$ & $4,5 \mathrm{mg}$ Suprarenin sub- \\
10 & $15-10$ & 45 & 0,5 & nicht bestimmt & - & kntan. & \\
10 & $45-11$ & 15 & 2,5 & $0,99(?)$ & + & & \\
11 & $15-11$ & 45 & 3,5 & 0,29 & + & & \\
11 & $45-12$ & 15 & 3,5 & Spur & + & & \\
12 & $15-2$ & 00 & 11,0 & Spur & + & & \\
2 & $00-3$ & 00 & 8,0 & Spur & + & & \\
9 & $00-4$ & 00 & 4,0 & Spur & + & & \\
4 & $00-5$ & 00 & 3,0 & Spur & + &
\end{tabular}

6. Kaninchen, männlich, 3000 g. 8. Mai 1906.

\begin{tabular}{|c|c|c|c|c|}
\hline $1100-1200$ & 1,0 & 0,409 & - & $12^{\text {h }} 6 \mathrm{mg}$ Suprarenin subkutau. \\
\hline $1200-1285$ & 6,0 & 0,35 & + & Von 11 h 5 ' Auftreten eines braunen \\
\hline $1235-100$ & 7,0 & 0,194 & + & Farbstoffes im Urin, der auf $\mathrm{Zu}-$ \\
\hline $100-200$ & 16,0 & 0,257 & + & satz von Alkali abblasst. \\
\hline $200-300$ & 14,0 & 0,164 & + & \\
\hline $300-400$ & 11,0 & 0,164 & + & \\
\hline $400-500$ & 8,5 & 0,105 & & \\
\hline $500-600$ & 11,5 & 0,292 & + & $6 \mathrm{~h}$ noch ca. $3 \mathrm{ccm}$ daneben gelaufen. \\
\hline
\end{tabular}


7. Kaninchen, männlich, 2400 g. 15. Mai 1906.

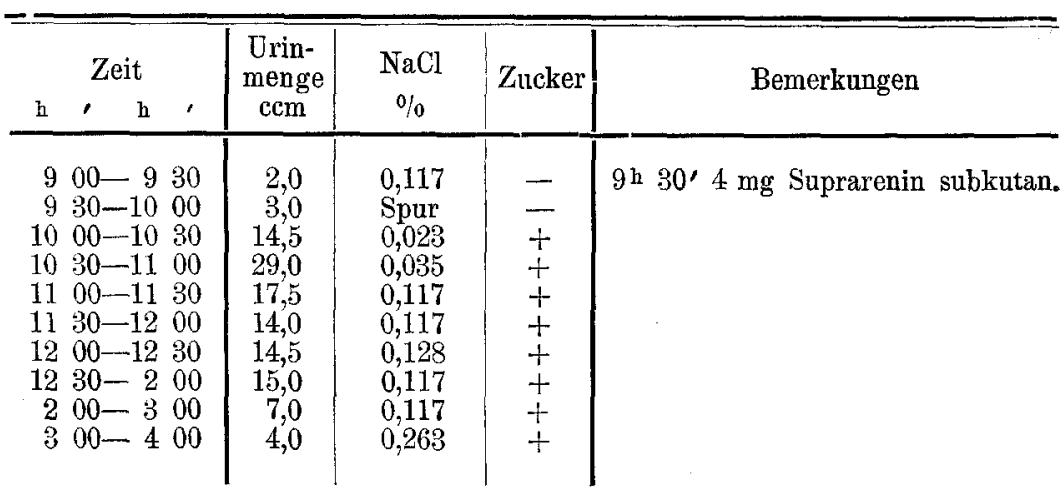

8. Kaninchen, männlich, $3200 \mathrm{~g}$. 10. Mai 1906 auf Reis- und Wasserfutter gesetzt. 11. Mai Urin $=0,584 \% \mathrm{NaCl}$. 16. Mai Urin $=0,26 \% \mathrm{NaCl}$.

\begin{tabular}{|c|c|c|c|c|}
\hline $\begin{array}{l}\text { 17. Mai } \\
930\end{array}$ & nicht best. & 0,012 & $\ldots$ & $9 \mathrm{~h} 30^{\prime}$ katheterisiert: $6.5 \mathrm{mg}$ \\
\hline $930-1010$ & 2,0 & Spur & - & prarenin. hydrochlor. sublatan. \\
\hline $1010-10 \quad 30$ & 2,0 & 1 & - & \\
\hline $1030-1100$ & 8,5 & $"$ & - & \\
\hline $1 \quad 00-11 \quad 30$ & 4,4 & & - & \\
\hline $1130-12 \quad 10$ & 6,3 & 0,058 & + & \\
\hline $12 \quad 10-1240$ & 4,5 & 0,058 & + & $12^{\mathrm{h}} 40^{\prime}$ nochmals $6 \mathrm{mg}$ Suprar. \\
\hline $1240-200$ & 6,0 & 0,117 & + & hydrochlor. subkutan. - Der u \\
\hline $200-300$ & 8,0 & 0,058 & + & $2^{\mathrm{h}}$ erhaltene Urin blutig. \\
\hline $300-415$ & 7,0 & 0,058 & + & \\
\hline 41 & 4,0 & 0,087 & & \\
\hline $515-600$ & 2,0 & & - & Urin stark blutig. \\
\hline
\end{tabular}

9. Hund, männlich, 13000 g. 28. Mai 1906.

\begin{tabular}{rrr|l|l|l|l}
10 & $30-11$ & 15 & 6,5 & 0,379 & - & $10^{\mathrm{h}} 30^{\prime} 0,08 \mathrm{mg}$ Morphin subku- \\
11 & $15-11$ & 45 & 6,0 & 1,226 & - & $\tan ; 11 \mathrm{~h} 15^{\prime}$ 10 mg Suprarenin. \\
11 & $45-12$ & 15 & 2,0 & 0,467 & - & \\
12 & $15-1$ & 00 & 3,0 & 0,409 & - & \\
1 & $00-2$ & 00 & 5,0 & 0,204 & - & \\
2 & $00-4$ & 00 & 9,5 & 0,058 & - & \\
4 & $00-5$ & 00 & 3,5 & 0,029 & - &
\end{tabular}

10. Derselbe Hund; kein Morphin. 11. Juni 1906.

\begin{tabular}{|c|c|c|c|c|}
\hline $950-1020$ & 3,5 & 0,2 & - & $10^{\text {h }} 20^{\prime} 5 \mathrm{mg}$ Suprarenin subkutan \\
\hline $1020-1050$ & 0,9 & $0,19(?)$ & - & \\
\hline $1050-1120$ & 3,5 & 0,44 & - & \\
\hline $11 \quad 20-12 \quad 20$ & 5,0 & 0,29 & - & \\
\hline $1220-130$ & 8,0 & 0,024 & + & \\
\hline $130-300$ & 14,0 & schwache & + & \\
\hline $300-430$ & 9,0 & do. & + & \\
\hline $430-5$ & 3,0 & do. & jiht dentl. & \\
\hline
\end{tabular}


Joh. Biberfeld:

11. Kaninchen, $1900 \mathrm{~g}$; schreibt seinen Blutdruck am Kymographion 13. Oktober 1906.

\begin{tabular}{|c|c|c|c|c|c|c|c|}
\hline h & $\begin{array}{r}\text { Zeit } \\
, \quad \mathbf{h} \\
\end{array}$ & 1 & $\begin{array}{l}\text { Urin- } \\
\text { menge } \\
\text { cem }\end{array}$ & $\begin{array}{c}\mathrm{NaCl} \\
\%\end{array}$ & Zucker & $\begin{array}{l}\text { Blutdruck } \\
\mathrm{mm} \mathrm{Hg}\end{array}$ & Bemerkungen \\
\hline $\begin{array}{l}10 \\
10 \\
11\end{array}$ & $\begin{array}{l}20-10 \\
50-11 \\
20-11\end{array}$ & $\begin{array}{l}50 \\
20 \\
50\end{array}$ & $\begin{array}{l}1,1 \\
3,0 \\
5,0\end{array}$ & $\begin{array}{l}0,26 \\
0,35 \\
0,76\end{array}$ & $\overline{+}$ & $\begin{array}{ll}10 \text { h } 50^{\prime} & 42,0 \\
11 \text { h } 13^{\prime} & 46,0 \\
11 \text { h } 40^{\prime} & 35,0\end{array}$ & \multirow[t]{5}{*}{$\begin{array}{l}10 \mathrm{~h} 50^{\prime} 5 \mathrm{mg} \text { Suprarenin } \\
\text { subkutan. }\end{array}$} \\
\hline 11 & $50-12$ & 40 & 4,5 & 0,88 & +\{ & $\begin{array}{ll}12 \text { h } 10^{\prime} & 38,0 \\
12^{\text {h }} 40^{\prime} & 40,0\end{array}$ & \\
\hline 12 & $40-1$ & & 3,3 & 0,98 & + & $1^{\text {h h }} 00^{\prime} \quad 39,0$ & \\
\hline 1 & $30-2$ & & 2,1 & 0,76 & + & Ib $30^{\prime} \quad 40,0$ & \\
\hline 2 & $30-4$ & & 1,8 & 0,41 & -\{ & $\begin{array}{ll}2 \mathrm{~h} 50^{\prime} & 21,0 \\
3 \mathrm{~h} 30^{\prime} & 24,5\end{array}$ & \\
\hline
\end{tabular}

12. Kaninchen, $1800 \mathrm{~g}$, am Kymographion. 27. Oktober 1906.

\begin{tabular}{|c|c|c|c|c|c|}
\hline $1100-1130$ & 1,9 & 1,11 & - & $11^{\mathrm{h}} 30^{\prime} \quad 43,0$ & $11^{\text {h }} 30^{\prime} 2 \mathrm{mg}$ Suprarenin \\
\hline $1130-1200$ & 2,2 & 1,24 & - & $\begin{array}{ll}11 \text { h } 50^{\prime} & 45,0 \\
11 \text { h } 52^{\prime} & 43,0 \\
12 \text { h } 00^{\prime} & 45,0 \\
12 \text { h } 10^{\prime} & 41,5\end{array}$ & $\begin{array}{l}\text { liche Vaguspulse; } 12^{\mathrm{h}} \\
\text { starke Abnahme der Puls- } \\
\text { frequezz. }\end{array}$ \\
\hline
\end{tabular}

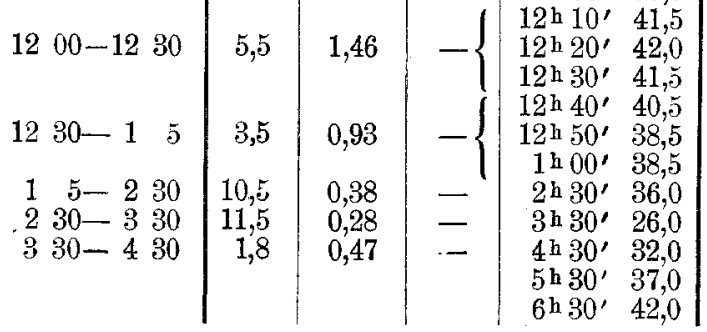

13. Kaninchen, $1700 \mathrm{~g}$, am Kymographion. 31. Oktober 1906.

\begin{tabular}{|c|c|c|c|c|c|}
\hline $1000-1035$ & 1,5 & 0,53 & - & 10 h $00^{\prime} \quad 48,0$ & $10^{\mathrm{h}} 40^{\prime} 2 \mathrm{mg}$ Suprarenin \\
\hline $1035-11 \quad 5$ & 6,0 & 0,75 & - & $\begin{array}{ll}10^{\mathrm{h}} 40^{\prime} & 44,5 \\
10^{\mathrm{h}} 55^{\prime} & 42,5\end{array}$ & $\begin{array}{l}\text { subkutan; } 11 \text { h } 25^{\prime} 2 \mathrm{mg} \\
\text { Suprarenin subkutan. }\end{array}$ \\
\hline $115-1135$ & 3,4 & 1,5 & & 11 h $20^{\prime} 41,0$ & \\
\hline $1135-1210$ & 3,2 & 0,93 & - & $12 \mathrm{~h} 00^{\prime} 40,0$ & \\
\hline $12 \quad 10-1245$ & 2,6 & 1,23 & & $\begin{aligned} 12 \mathrm{~h} 45^{\prime} & 45,0 \\
1 \mathrm{~h} 20, & 43,5\end{aligned}$ & \\
\hline $45-245$ & 21,1 & 0,6 & 一 & 1h $30^{\prime} \quad 49,5$ & \\
\hline $45-345$ & 6,0 & 0,64 & & & \\
\hline
\end{tabular}

14. Kaninchen, 2100 g. 1. November 1906 . Wird nur zum Katheterisieren aufgespannt, sonst im Käfig freigelassen.

\begin{tabular}{|c|c|c|c|c|c|c|}
\hline \multicolumn{3}{|c|}{ Zeit } & \multirow{2}{*}{$\begin{array}{c}\text { Urinmenge } \\
\text { ccm }\end{array}$} & \multirow{2}{*}{$\underset{\%}{\mathrm{NaCl}}$} & \multirow{2}{*}{ Zucker } & \multirow{2}{*}{ Bemerkungen } \\
\hline h & , h & , & & & & \\
\hline 9 & $45-10$ & 45 & 3,0 & 0,41 & - & $10^{\mathrm{h}} 45^{\prime} 4 \mathrm{mg}$ Suprarenin \\
\hline 10 & $45-11$ & 15 & 5,5 & 0,46 & - & subkutan. \\
\hline 11 & $15-11$ & 45 & 28,0 & 0,35 & $\ldots$ & \\
\hline 11 & $45-12$ & 15 & 17,0 & 0,41 & + & \\
\hline 12 & $15-12$ & 45 & 11,0 & 0,32 & + & \\
\hline 12 & $45-2$ & 00 & 14,2 & 0,15 & + & \\
\hline 2 & $00-\overline{3}$ & 00 & 4,1 & Spur & + & \\
\hline 3 & $00-4$ & 00 & 4,5 & Spur & + & \\
\hline 4 & $00-5$ & 00 & 3,5 & 0,15 & + & \\
\hline$\Rightarrow$ & $00-6$ & & kein & rin 20 & alten & \\
\hline
\end{tabular}


15. Kaninchen, 2000 g. 2. November 1906. Wird während des ganzen Versuches aufgebunden im Thermostaten $\left(24^{\circ} \mathrm{C}\right)$ gehalten; nur zum Katheterisieren wird es herausgenommen.

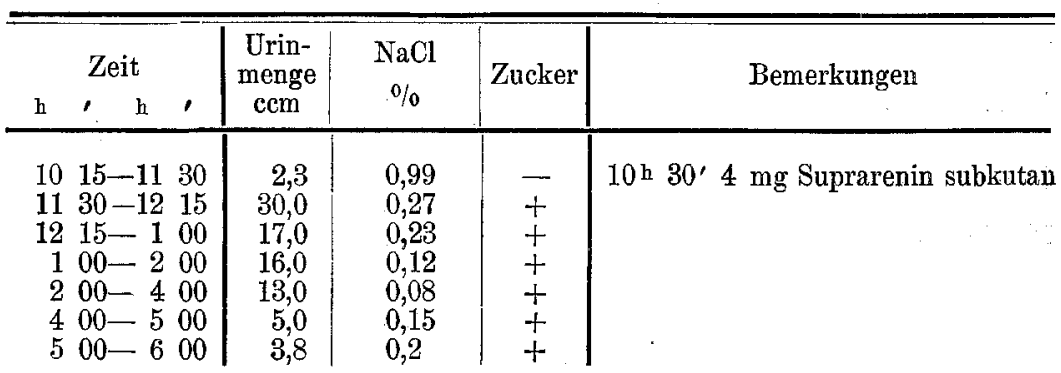

16. Kaninchen, 2900 g. Während des ganzen Versuches aufgebunden gehalten und nur notdürftig zugedeckt.

\begin{tabular}{|c|c|c|c|c|}
\hline $1000--1100$ & 5,7 & 0,23 & $1-$ & 11 h $5 \mathrm{mg}$ Suprarenin subkutan \\
\hline $\begin{array}{lll}11 & 00-11 & 30 \\
11 & 30-12 & 00\end{array}$ & kein & Urin zu & erhalten & \\
\hline $\begin{array}{lll}11 & 30-12 & 00 \\
12 & 00-12 & 45\end{array}$ & $\begin{array}{l}0,0,0 \\
0,0\end{array}$ & $\begin{array}{l}0,00 \\
0,03\end{array}$ & + & \\
\hline $1245-130$ & 36,0 & 0,05 & + & \\
\hline $130-230$ & 10,2 & 0,08 & + & \\
\hline $230-330$ & 25,0 & 0,08 & + & \\
\hline $330-440$ & 20,5 & 0,12 & + & \\
\hline
\end{tabular}

17. Hund, männlich, 8500 g. 6. März 1907.

\begin{tabular}{|c|c|c|c|c|}
\hline $1100-1130$ & 5 & Analyso rer- & - & $\begin{array}{l}11 \mathrm{~h} 0,03 \text { Morphin subkut.; } 11^{\text {h }} 30^{\prime} \\
10 \mathrm{mg} \text { Suprarenin ( } 1 \% \text { Lösung })\end{array}$ \\
\hline $1130-1200$ & 3,5 & 0,26 & - & \\
\hline $1200-1230$ & 5,0 & 0,26 & - & \\
\hline $1230-100$ & 3,0 & 0.17 & & \\
\hline $100-200$ & 3,2 & & & \\
\hline $\begin{array}{lll}2 & 00-3 & 00 \\
3 & 00-4 & 00\end{array}$ & 5,0 & 0,17 & - & \\
\hline $400-500$ & 2,6 & 0,17 & $+(?)$ & \\
\hline
\end{tabular}

18. Kaninchen, $1900 \mathrm{~g}$; am Kymographion. 7. März 1907.

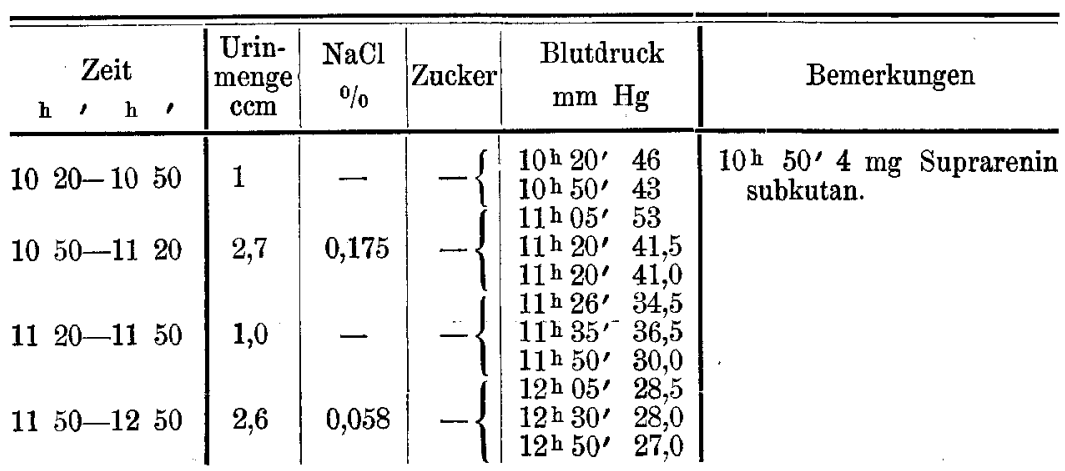

Um $1^{\mathrm{h}} 30^{\prime}$ Tod; kein Urin in der Blase; Lungenödem. 
19. Kaninchen, $2400 \mathrm{~g}$, am Kymographion. 8. März 1907.

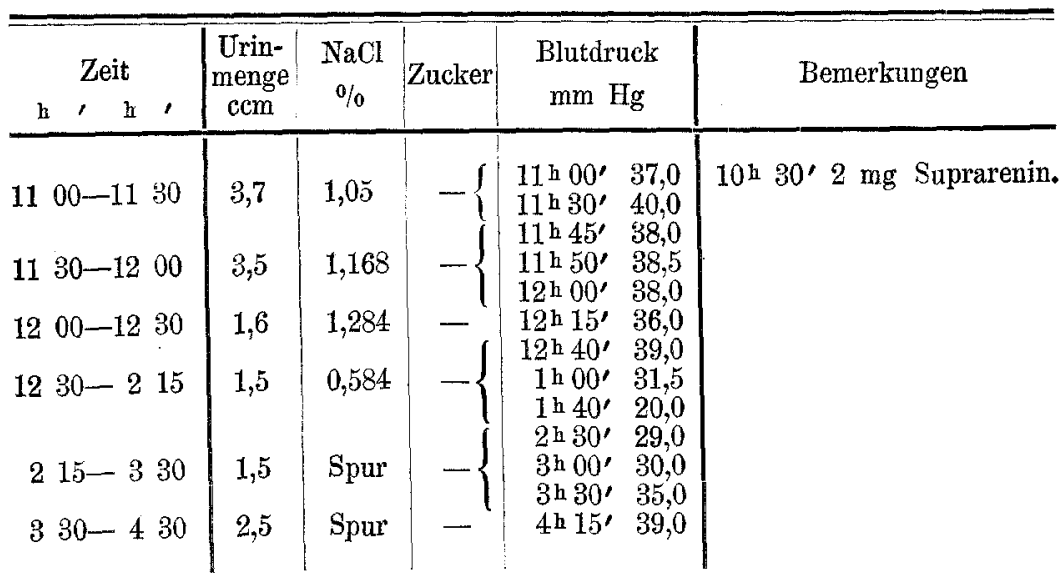

20. Hund von Versuch $17 \mathrm{~g}$; kein Morphin. 11. März 1907.

\begin{tabular}{|c|c|c|c|c|c|c|}
\hline $\mathbf{h}$ & $\begin{array}{l}\text { Zeit } \\
, \quad h\end{array}$ & ' & $\begin{array}{l}\text { Urin- } \\
\text { menge } \\
\text { ccm }\end{array}$ & $\begin{array}{c}\mathrm{NaCl} \\
\%\end{array}$ & Zucker & Bemerkungen \\
\hline $\begin{array}{r}9 \\
9 \\
10 \\
10 \\
11 \\
11 \\
12 \\
1 \\
2 \\
4\end{array}$ & $\begin{array}{ll}20-9 & 5 \\
50-10 & 2 \\
20-10 & 5 \\
50-11 & 2 \\
20-11 & 5 \\
50-12 & 5 \\
50-1 & 5 \\
50-2 & 5 \\
55-4 & 0 \\
00-5 & 0\end{array}$ & $\begin{array}{l}0 \\
0 \\
0 \\
0 \\
0 \\
0 \\
6 \\
5 \\
5 \\
0 \\
0\end{array}$ & $\begin{array}{l}24 \\
13 \\
21 \\
16,5 \\
21,0 \\
29,0 \\
26,0 \\
25,0 \\
10,4 \\
8,0\end{array}$ & $\begin{array}{l}\overline{1,17} \\
1,12 \\
1,05 \\
1,109 \\
0,996 \\
0,98 \\
0,87 \\
0,7 \\
0,81\end{array}$ & $\begin{array}{l}- \\
- \\
- \\
\overline{+} \\
+ \\
+ \\
+ \\
+\end{array}$ & $\begin{array}{l}\text { Blase war vorher nicht ganz entleert } \\
\text { worden. } 10^{\mathrm{h}} 20^{\prime} 5 \mathrm{mg} \text { Suprar. } \\
\text { subkutan. }\end{array}$ \\
\hline
\end{tabular}

21. Kaninchen mit $2900 \mathrm{~g}$. 15. März 1907. $11^{\mathrm{h}} 30^{\prime}$ katheterisiert, Katheter bleibt bis $12^{\mathrm{h}}$ liegen; $11^{\mathrm{h}} 55^{\prime}$ 0,04 Morphin, $12^{\mathrm{h}} 4 \mathrm{mg}$ Suprarenin subkutan.

\begin{tabular}{rrr|r|l|l|}
11 & $30-12$ & 00 & 1,5 & 0,876 & - \\
12 & $00-12$ & 35 & 3,5 & 0,35 & - \\
12 & $35-1$ & 00 & 1,6 & 0,23 & - \\
1 & $00-2$ & 10 & 4,5 & 0,118 & + \\
2 & $10-3$ & 00 & 8,5 & 0,118 & + \\
3 & $00-4$ & 10 & 11,5 & 0,18 & + \\
4 & $10-5$ & 00 & 9,0 & 0,18 & + \\
5 & $00-6$ & 00 & 3,5 & 0,24 & +
\end{tabular}

